\title{
Ein Luftschloss aus Haselnüssen und anderen Lösungen
}

\section{Cristina Zimmermann}

Studierende der Medizin im 3. Jahr an der Universität Freiburg

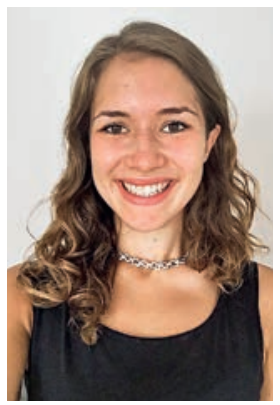

«So, jetzt kommt Herr M.!» Mit diesen Worten hat mich meine betreuende Hausärztin, Doktor B., schon die letzten 2 Tage auf die jeweiligen, kommenden Patienten vorbereitet. Doch auf was bereitet mich dieser Satz vor? Manchmal warten auf mich spannende Aufgaben, wie Fäden zu ziehen oder das erste Mal eine Fahrtauglichkeitsprüfung durchzuführen. Oft folgen auch Routineuntersuchungen, bei denen mit etlichen mir noch unbekannten Medikamentennamen um sich geworfen wird. Und hin und wieder folgt auch die Art von Gesprächen, bei denen man nicht so recht weiss, welcher Gesichtsausdruck denn nun passend ist. Es sind Gespräche, bei denen eine bodenständige Bäuerin gesteht, dass sie nicht mehr schlafen kann, weil sie ständig dieses "Tock» hört, mit dem ihr Bruder auf den Boden gefallen und verstorben ist, oder der ruhige Ehemann einer Frau mit Hirnblutung unverhältnismässig laut ruft: "Es geht einfach nicht mehr!». Genau auf so ein Gespräch soll mich dieser vielseitig einsetzbare Satz diesmal vorbereiten.

Über ein Magazin gebeugt und mit seinem schwarzen Adidas-Trainingsanzug wirkt Herr M. etwas fremd in dem schicken, skandinavisch eingerichteten Wartezimmer. «Herr M.!» ruft Doktor B. freundlich in den Raum hinaus. Fast schon ertappt klappt Herr M. das Magazin zu und mustert mich skeptisch. Die Ärztin stellt mich wie jedes Mal vor und fragt, ob es denn in Ordnung sei, wenn ich dabei wäre. Zu meinem ehrlichen Erstaunen gibt sich Herr M. tolerant, meint, es müsse jeder ja einmal lernen, und zwingt sich ein Lächeln ab. "Zwingt» deshalb, weil man gleich sieht, dass das Lächeln nicht von innen heraus kommt. Es ist aber nicht schmerzverzerrt, wie man meinen könnte, nein, diesem Akt der Freundlichkeit fehlt die Echtheit, die Energie.

Noch während des Hinsetzens werfe ich einen Blick auf den Computerbildschirm. Depression, Sarkoidose, Herzinfarkt, Vorhofflimmern, so steht es in seiner Patientenakte. Diese Diagnose mit dem Mann mit Dreitagebart und leerem Blick auf dem Stuhl gegenüber von Doktor B. zu vereinbaren ist nicht schwer. Mit welcher Energie er dann aber den Grund seines Besuches schildert, erstaunt mich. Er brauche ein Langzeit-
Zeugnis für die Invaliden-Behörde. Seine Psychologin hat ihm dieses anscheinend nicht ausstellen wollen, da er zu einigen Terminen nicht erschienen ist. Herr M. schiesst gleich mit seiner Meinung heraus: Dies bestätige doch erst recht, wie schlecht es ihm ginge. Er sei wirklich enttäuscht von seiner Psychologin. Seine teilweise hastige Gestikulation mit den Händen verleiht seiner Bestürzung eine seltsame Gewichtung: Man spürt heraus, dass Herr M. wahrscheinlich schon einige Nächte zu viel über das Statement der Psychologin nachgedacht hat. Der weiteren Ausführung der Gründe seiner Arbeitsunfähigkeit, kann Doktor B. nicht mehr als ein paar zustimmende «Jaja» hinzufügen. Mehr hätte Herr M. aber auch nicht zugelassen. Das hätte ihn nur in seinem Fluss der Entrüstung unterbrochen. Als ob Herr M spüren würde, dass Doktor B. und ich seine Empörung über seine Psychologin als eigene Unsicherheit und Unzulänglichkeit abgetan haben, ändert er seinen Monolog in einen Dialog mit sich selbst. Eigentlich sei die Psychologin doch nicht so schlecht, aber er brauche doch eine festere Hand. Sie sei so nett, aber er brauche mehr Druck. Das Ergebnis seines fünfminütigen Gesprächs mit sich selbst ist, dass er seiner jetzigen Psychologin noch eine Chance gibt.

Sich seines Zeugnisses sicher und seines Frustes erleichtert, lässt Herr M nun schliesslich doch Doktor B. zu Wort kommen. Schnell wird das Gespräch auf seinen körperlichen Gesundheitszustand gelenkt. Herr M. beginnt ein Bild zu malen von einem vitalen Patienten, der täglich zwei bis drei Stunden laufen geht, regelmässig schwimmt, sich sehr gesund ernährt und natürlich keinen Alkohol trinkt. Ein Bild, das den Anschein macht, ihn durch die Erzählung davon schon zu erschöpfen. Doktor B. gibt sich trotzdem zufrieden. Was sollte sie auch anderes tun? Ihm ins Gesicht sagen, dass sie beide wüssten, dass es wohl kein einziger Ziegel seines gebauten Luftschlosses in die Realität schaffen würde? Nein, das kann sie weder Herrn M. noch sich antun. Mit einem wütenden Patienten umzugehen ist leicht, zustimmend mit dem Kopf zu nicken ist leicht, aber das was nach dem Zusammenbruch seines Luftschlosses passieren 
würde, wäre es nicht. Also sitzen beide da und reden über einen Patienten, der Herr M. gerne wäre und Doktor B. gerne hätte.

Um von den eindringlichen Blicken von Herrn $M$. zumindest für einen kurzen Moment erlöst zu werden, flüchtet sich Doktor B. in dessen Patientenakte. Erstaunlich, was für eine beruhigende Wirkung eine Tabelle mit Blutwerten haben kann, in ihnen findet sie Sicherheit. Hämoglobin-, Cholesterin- und Leukozyten-Werte sind ihr tägliches Brot. Man kommt zu ihr als erfahrene Hausärztin, um genau solche Dinge zu besprechen und meist nicht viel mehr. Die Gespräche bleiben oft oberflächlich: Auf das obligatorische «Hallo, geht es Ihnen gut?» wird eigentlich fast ein «Ja» erwartet. Ein «Mir schmerzt der Arm schon wieder», oder ein "Ja danke mir schon, aber meine Kinder sind krank», sind sicher nicht unüblich, aber für eine ehrliche, ausführlichere Antwort gibt es weder im Terminkalender einer ausgelasteten Arztpraxis noch im Budget der Krankenkassen oder im Alltag der Patienten wirklich Platz. Dann gibt es allerdings auch wieder Menschen, die einen Besuch bei ihrem Hausarzt nutzen um genau diese Frage endlich ehrlich beantworten zu können. Sie glauben bei einem Arzt an der richtigen Adresse zu sein. Der wird wissen wie damit umzugehen ist, im Gegensatz zu allen anderen. Aber dass man, gerade weil man jeden Tag so viele Menschen, so viele Schicksale sieht, überfordert sein kann, passt für die wenigsten in das Bild des Gottes in Weiss.

Nun sehe ich Doktor B., der in diesem Moment nicht nur der weisse Kittel, sondern auch die Sicherheit fehlt. Sie findet sie wieder im zu hohen Cholesterinwert. Die passende Frage hat Doktor B. schnell gestellt, sie befindet sich wieder auf bekanntem Terrain. Herr M. meint mit einem ernsten und eindringlichen Gesichtsausdruck, er wisse nun endlich wie er dieses Problem in den Griff bekäme: Haselnüsse. Er habe es gegoogelt. So werde alles gut, ganz sicher, er merke es schon. Was er genau mit "alles» meinte, weiss ich nicht. Seine eigentlichen Medikamente habe er übrigens selbständig abgesetzt. Genauso mit dem Blutdruckmedikament: Er habe seine Dosis von einer Tablette pro Tag «anhand seiner Erfahrungswerte manipuliert», also halbiert. Während ich noch über seine interessante Wortwahl nachdenke, traut sich Doktor B nun bei diesem Thema mehr einzuschreiten. Sein Blutdruck sei wirklich $\mathrm{zu}$ hoch, er solle wieder ein ganze Tablette nehmen. In ihrer Stimme liegt eine Bestimmtheit, die ich, seit Herrn $M$ das Zimmer betreten hat, nicht mehr gehört habe. Offenbar beeindruckt dies auch Herrn M, sodass er mit überschwänglicher Begeisterung, wie ich sie noch selten ursächlich einer Tablette gesehen habe, zustimmt.

Und so kommt es, dass Herr $\mathrm{M}$ wie jeder andere Patient mit einer Packung blutdrucksenkenden Medikamente in der Hand das Sprechzimmer verlässt. Während des Hinausgehens beobachte ich ihn, wie er noch kurz an dem Ständer mit Infobroschüren über alle möglichen Behandlungen stehen bleibt, zufrieden lächelt und sich ein Exemplar mitnimmt. Vielleicht sind das ja seine neuen Haselnüsse.

Doktor B. schliesst währenddessen die Patientenakte von Herrn M. und macht das Zimmer für den nächsten Patienten bereit. Wir sind spät dran: 24 Minuten statt 20 Minuten.

«So jetzt kommt Frau F.!»

\section{Medizinischer Bericht}

Herr M., 56 Jahre, alleinstehend, bezieht Invalidenrente und beschreibt sich selbst als sehr aktiv. Diagnose Depression gestellt. Ist vorstellig, da er ein Langzeitzeugnis benötigt und Beschwer den über seine momentane Psychotherapeutin vorzubringen hat. Zudem Besprechung über die Einstellung seiner Hypertonie und seiner Hypercholesterinämie: Der Patient wehrt sich gegen medikamentöse Behandlung.

Ausserdem: Zustand nach Sarkoidose und Myokardinfarkt, latentes Vorhofflimmern und mit CPAP behandelte Schlafapnoe. 